\title{
Steady-State Optimal Insulin Infusion for Hyperglycemic ICU Patients
}

\author{
J. Geoffrey Chase, Graeme C. Wake, Z-H Lam, J-Y Lee, K-S Hwang and G. Shaw \\ Dept of Mechanical Engineering and Dept of Mathematics \& Statistics \\ University of Canterbury \\ Private Bag 4800 \\ Christchurch, New Zealand \\ Email: g.chase@mech.canterbury.ac.nz
}

\begin{abstract}
Close control of blood glucose levels significantly reduces vascular complications in diabetes, as well as having beneficial effect for cardiac and other critical care patients. Recent studies have shown that tight regulation of blood glucose level in intensive care unit patients can reduce mortality by as much as $45 \%$. This paper presents an optimal insulin infusion algorithm to tightly regulate blood glucose for ICU and other critical care patients when the glucose input is known. Results show an ideal, flat blood glucose response when subjected to a typical ICU glucose input as well as for a far more extreme case.
\end{abstract}

\section{Introduction}

Hyperglycemia and insulin resistance are common in critically ill patients, even if they have not previously had diabetes. The normalization of blood glucose levels with insulin therapy to far tighter levels than normally employed has recently been seen to improve the mortality of ICU patients by as much as 45\% [1]. Specifically, the use of a 4-hour bandwidth blood glucose monitoring and insulin therapy regime has been found to reduce mortality in critical care patients. The methodology restricted blood glucose levels to approximately $5.8 \mathrm{mmol} / \mathrm{L}$, the maximum level typically desired by diabetes clinicians. Normal critical care blood glucose management will often allow for levels two times this value, with potentially increased patient risk.

Ambulatory diabetic individuals monitor food intake and daily activity to maintain blood sugar levels at an adequate level. In the ICU case patients are often hyperglycemic with no previous history, due to the stress the entire bodily system is undergoing. As a result, this condition can be far different from an ambulatory diabetic individual as ICU feeding, while variable in some cases, provides a known glucose input profile to a patient with a measurable insulin resistance. However, in each case high blood glucose levels must be tightly regulated using insulin therapy.

Though devices that can measure glucose level and administer insulin exist, they do just that - measure and inject - with no automated interface between the two. ICU patients typically have their blood glucose monitored 2-6 times per day and are treated with insulin by a standard protocol to minimize blood glucose excursions.

Most commonly available glucose sensing devices operate by measuring the blood glucose content of a small finger-prick blood sample, an irritating method upon frequent use. However, recent advances in technology suitable for ICU patients has been developed that enable blood glucose measurement at rates from every 0.6-7.0 minutes [2-4]. With time constants on the order of minutes the faster of these rates are essentially continuous measurement. This rapid measurement enables feedback control, with knowledge of the glucose input, to be applied to minimize blood glucose levels toward the basal rate of 4.5-5.0 $\mathrm{mmol} / \mathrm{L}$, eliminating the exposure to elevated blood glucose levels and the damage that can result.

Typically, relaxed higher blood glucose levels are considered acceptable, as blood glucose management without automation does not typically deliver the data, or the ability to constantly modify insulin infusion rates to achieve tighter control. However, regular, automated blood glucose measurement provides the consistent volume of data necessary for such tight control, in contrast to the sometimes infrequent and inconsistent efforts available in busy critical care units. The ideal response would be a flat line at the basal level [5].

Whether it is in the area of understanding, modeling or managing diabetes $[6,7]$ years of research in this area has led to no shortage of potential solutions [8-17]. However, due to either the complexity of the proposed implementation, current technological limitations, models that are not physiologically verified, lack of required data, or the cost/complexity of realizing the results, these solutions are not yet fully practicable.

Several researchers have examined the analysis and automation of insulin as reviewed by Lehman et al. [18]. Many of the systems presented use control as a means of providing clinical advice or testing the efficacy of a new protocol [19-24]. A more complex, higher performance real-time control example uses model predictive control on a 19th order system identified model resulting in a $40 \%$ peak reduction and $23 \%$ reduction in settling time [25]. Optimal control using grid search theory, robust $\mathrm{H}$-infinity control, and 
variable structure controllers have also been studied, each using different models [11, 24-28]. However, the models used typically require either patient specific parameters that are not generically available and/or knowledge of glucose or exercise inputs that would not be known a priori. Finally, none study the impact of more frequent measurement enabled by recent advances in sensing technology or the potential for improved results in specialized, controlled cases such as the ICU.

This research develops the equations necessary for real-time steady-state optimal control of blood glucose excursions from the basal level. The control laws are derived from a basic model of the essential dynamics of the non-linear, multi-compartment glucoseregulatory system, and take advantage of the knowledge of the glucose input profile available in ICU and other critical care settings. The system is verified numerically and compared to normal, modeled human response to the same inputs, which recent studies have shown to be similar to PD slope control of blood glucose level [29].

\section{Dynamic System Model}

Comprehensive models, though they are very accurate in regimen evaluation, are generally unsuited for realtime control, requiring several time points of input to generate the insulin infusion profile. Additionally, they are not generic requiring patient-specific data and known glucose inputs. The aim of this research is to develop control schemes based on models that capture the essential system dynamics, do not require unavailable data, and are applicable to a wider variety of subjects. Simple models capture these essential dynamic behaviours, providing a more suitable foundation for real-time control design and analysis that can be applied to a broad range of patients.

A well known, and more importantly, physiologically verified model originated from the work of Bergmann et al. [15]. It utilises the concept of a remote compartment for the storage of insulin to account for the time delay between injection of insulin and its utilization to reduce blood glucose levels. Equations (1)-(3) show the equations used to define the system.

$$
\begin{aligned}
& \dot{G}=-p_{1} G-X\left(G+G_{B}\right)+P(t) \\
& \dot{X}=-p_{2} X+p_{3} I \\
& \dot{I}=-n\left(I+I_{B}\right)+u(t) / V_{I}
\end{aligned}
$$

Where $G$ is the plasma glucose concentration over basal $\left(\mathrm{mMol} \mathrm{L} \mathrm{L}^{-1}\right), G_{B}$ is the basal plasma glucose ( $\mathrm{mMol}$ $\left.L^{-1}\right), X$ is the generalized insulin variable for the remote compartment $\left(\mathrm{min}^{-1}\right), I$ is the insulin concentration above basal $\left(m U L^{-1}\right), I_{B}$ is the basal insulin level ( $m U$ $\left.L^{-1}\right), P(t)$ is the external glucose input $\left(\mathrm{mMol} \mathrm{L}^{-1} \mathrm{~min}^{-1}\right)$, $u(t)$ is the external insulin input $\left(m U L^{-1} \mathrm{~min}^{-1}\right), p_{3}$ is a patient parameter $\left(m U^{-1} L \mathrm{~min}^{-2}\right), V_{I}$ is the insulin distribution volume $(L)$, and $n, p_{1}, p_{2}$ are patient specific parameters $\left(\mathrm{min}^{-1}\right)$. The parameters, $p_{1}, p_{2}$ and $p_{3}$, may be changed to represent different conditions of the glucose regulatory system. For Type I diabetic individuals: $p_{1}=0, p_{2}=0.025, p_{3}=0.000013$ as obtained from medical research $[10,13,15]$.

The model is simple, yet accurately represents the essential dynamics of the human glucose regulatory system. The three equations represent insulin infusion dynamics, storage in a remote compartment, and glucose input and insulin utilization in a second compartment. The values of $n, V_{l}, G_{B}, I_{B}$ employed are defined, for an average-weighted male as: $V_{I}=12 \mathrm{~L}, n$ $=5 / 54 \mathrm{~min}^{-1}, G_{B}=4.5 \mathrm{mmol} \mathrm{L}{ }^{-1}, I_{B}=15 \mathrm{mU} \mathrm{L}^{-1}[10]$.

Most reported controllers use a simple feedback loop that employs the blood glucose level above basal, $G$, and more rarely its' derivative, $\dot{G}$, as sensor inputs, and the exogenous insulin infusion rate, $u(t)$, as the control output. These controllers measure the output from Equation (1) while directly influencing Equation (3) via the control action. In between these two equations is the time delay and dynamics of the remote compartment defined in Equation (2).

\section{Derivation of Optimal Control}

There are many complex influences between glucose and insulin concentration for any person, normal or diabetic. However, the steady state glucose concentration in the body is finally a function of how much insulin is present. The goal is to minimise excess glucose, $G$, and its rate of change, $\dot{G}$, and ensure that excursions from the basal value, $G_{b}$, are minimised in magnitude and duration with no hypoglycemic overshoot below the basal level. Ideally, blood sugar levels should be maintained in a tight range around the basal level, varying with glucose input, or feeding, level. This approach trends toward the concept that the ideal blood glucose curve should be relatively, if not completely, flat at $G(t)=0$.

The optimal solution for $u(t)$ can be obtained analytically for the steady-state case where $\dot{G}=0$ is the desired condition that allows the optimal $u(t)$ that results in $G=0$ to be determined.

First, Equation (3) is solved and the exact solution for $I(t)$ determined in terms of the exogenous insulin infusion $u(t)$.

$$
I(t)=I_{B}\left(e^{-n t}-1\right)+\frac{e^{-n t}}{V_{I}} \int_{0}^{t} e^{n t^{\prime}} u\left(t^{\prime}\right) d t^{\prime}
$$


Similarly, using Equation (2), the solution for $X(t)$ is obtained in terms of $I(t)$ and, using Equation (4), directly expressed in terms of $u(t)$.

$$
\begin{aligned}
X(t)= & \frac{p_{3} I_{B}}{p_{2}-n}\left(e^{-n t}-e^{-p_{2} t}\right)+\frac{p_{3} I_{B}}{p_{2}}\left(e^{-p_{2} t}-1\right)+ \\
& \frac{p_{3}}{V_{I}\left(p_{2}-n\right)} \int_{0}^{t} u\left(t^{\prime}\right)\left\{e^{-n\left(t-t^{\prime}\right)}-e^{-p_{2}\left(t-t^{\prime}\right)}\right\} d t^{\prime}
\end{aligned}
$$

Since the goal is to minimize $G$ to approach or equal zero, the assumption of $G=\dot{G}=0$ at steady state is applied to Equation (1) to obtain a steady state optimal solution, from the remaining terms:

$$
P(t)=G_{B} X(t)
$$

More specifically, setting $\dot{G}=0$ is a means of obtaining the optimal solution for $u(t)$ to obtain any desired function or value of $G$. Since we desire, in the steady state that $G=0$, the resulting exogenous insulin input, $u(t)$, can be determined from Equation (6), using the expression for $X(t)$ in terms of $u(t)$ from Equation (5). Note that if the patient is a diabetic and $p_{1}=0$, the same result is obtained.

Substituting Equation (5) into Equation (6) and using the Laplace transform and its inverse, to simplify the convolution integrals and algebra, the optimal steady state exogenous insulin infusion function solution for $u(t)$ is obtained:

$$
\begin{aligned}
& u(t)=\frac{V}{p_{3} G_{b}} \bar{P}+u_{0} \\
& \bar{P}=\ddot{P}(t)+\dot{P}(0)+\left(n+p_{2}\right)(\dot{P}(t)+P(0))+n p_{2} P(t)
\end{aligned}
$$

The optimal solution includes first and second derivatives of the exogenous glucose input $P(t)$, as might be expected in solving a series of two first order differential equations to obtain the algebraic solution the third equation in Equation (6). However, it is unrealistic to implement in cases where $P(t)$ is not known a priori. Since $u(t)$ can become negative it may be necessary to infuse additional glucose of the first or second derivatives of $P(t)$ are significantly negative in value. Equation (7) is also an explicit function of the time constants and other model parameters subjecting it to potential modelling error. This solution does act as a benchmark for the performance of other controllers, as well as being suitable for cases where the glucose input is well known and controlled, such as in critical care and other managed care settings.

Note that the steady-state insulin infusion without exogenous glucose input, $u(t)=n V_{I} I_{B}=u_{0}$, corresponds to the result obtained from Equation (7) if $P(t)=0$. Since $u_{0}$ is the basal infusion rate of insulin in the absence of glucose input this result serves as a basic, but essential, check on the optimal control law derived. More specifically, it implies that the optimal control law reverts to the basal infusion when there is no exogenous glucose input, as should be expected.

This optimal solution will provide an optimal insulin infusion profile for any case where the initial conditions match the steady state assumptions. It also does not employ blood sugar feedback, being a closed form solution with knowledge of the glucose input, as most previously reported literature. However, knowledge of the input limits the solution to specialized cases, such as the ICU.

\section{Numerical Tests and Verification}

\subsection{Slow Infusion of Glucose}

Feeding protocols for ICU patients vary by country and region. However, the typical feeding protocol typically consists of a very low number of calories being slowly infused. The infusion is either (nearly) continuous, or given over a 1-3 hour period, 2-4 times daily.

Figure 1 shows the glucose response for a 400 calorie input infused gradually over 150 minutes for both a normal, non-diabetic response and for the optimal infusion for a diabetic individual, which is an ideal flat line. Note that the optimal result is not truly flat and $G(t)$ varies between $G=+/-0.01 \mathrm{mmol} / \mathrm{L}$, as shown in Figure 2. Figure 3 shows the insulin input profiles for both cases. Note that the optimal insulin infusion profile is essentially an injection at the first part of the feeding period and that the area under the optimal and normal curves, representing the total insulin infused, is approximately the same.

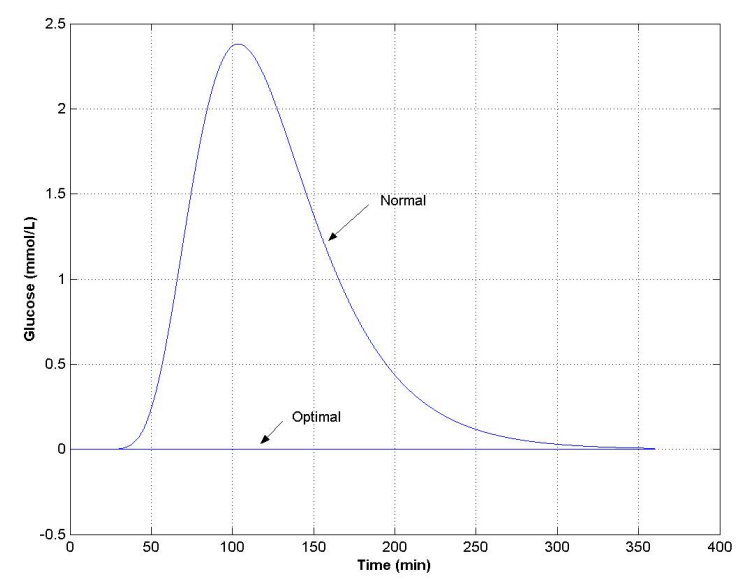

Figure 1: Glucose response to slow infusion. 


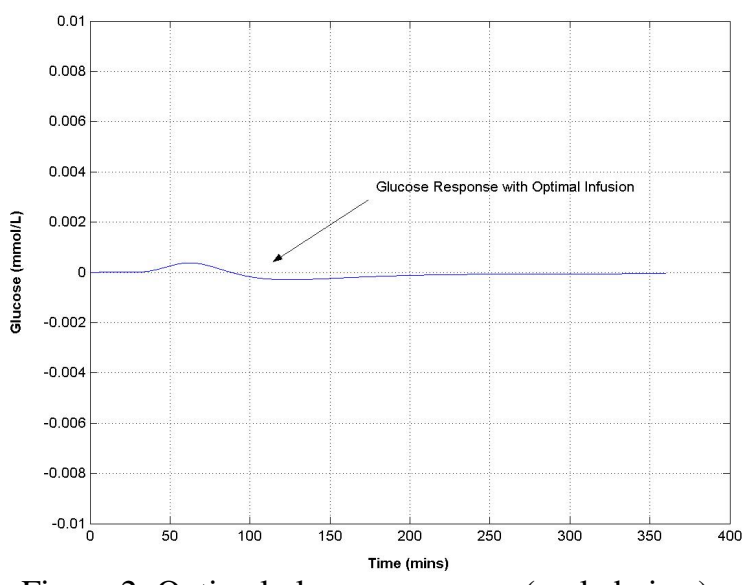

Figure 2: Optimal glucose response (scaled view).

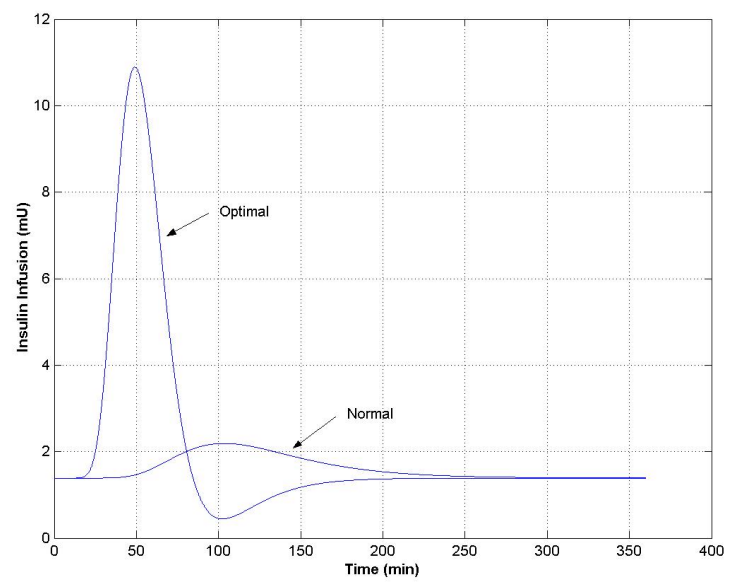

Figure 3: Insulin response to slow infusion.

\subsection{Multiple Meal Glucose Input}

The oral glucose tolerance test (OGTT) is a large step input test of the glucose regulatory system often performed to diagnose diabetes. A fasting subject consumes 400-800 calories of glucose and the response is observed. The OGTT input may be modelled by using the lognormal distribution defined in Equation (8).

$$
P(t)=P_{m} e^{-a(\ln (b t)-c)^{2}}
$$

Where $P_{m}$ is the peak value and $a, b$ and $c$ are constants, which determine the slopes and curvature.

A multiple meal glucose input can be constructed from this form of input. The specific input is designed to be extreme and its inputs vary in magnitude from 50-400 calories in two groups, at $t=0,10,30$ minutes and at $t=$ 210 and 300 minutes. At the end of 6 hours, the total intake of glucose into the body is over 1000 calories with 1000 calories input over the first 4 hours.

As seen in Figure 4, the input profile provides an extreme test with large variations over time that are significantly greater than what is typically encountered in an ICU setting to provide a very rigorous test.

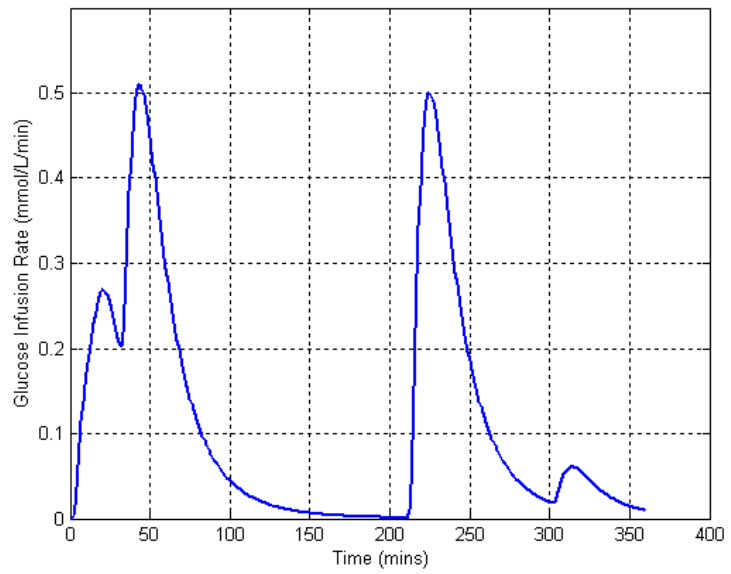

Figure 4: Multi-meal glucose input profile.

The response of the optimal controller to this system as well as the modelled normal human response is shown in Figures 5 and 6 . Figure 5 presents the glucose response over basal level, $G$, for normal, non-diabetic and optimally controlled diabetic individuals. Figure 6 shows the insulin infusion rate for the same cases.

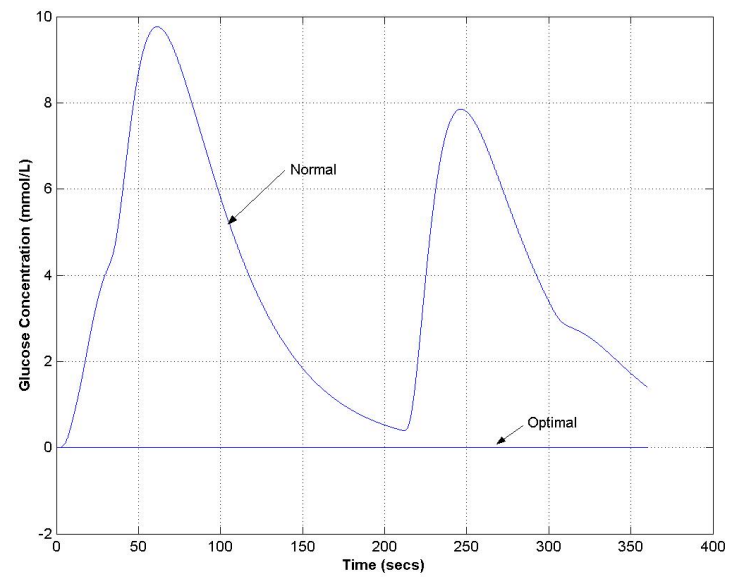

Figure 5: Glucose response to large multi-meal input.

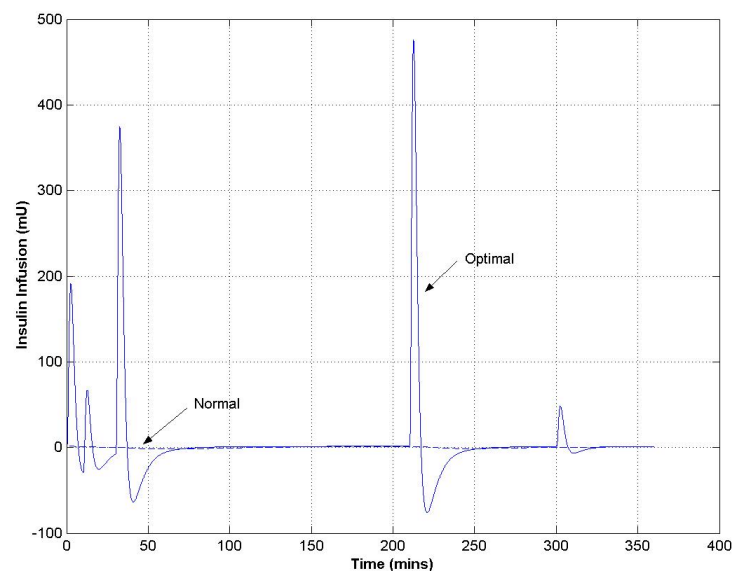

Figure 6: Insulin response for multi-meal input. 
Figure 5 has the same essentially flat glucose response for the optimal case, as it did for the slow infusion. However, the cost of obtaining this response is seen in Figure 6, where the insulin response for the optimal case consists of steep, sudden infusions that mimic injection profiles as each feeding infusion starts. However, due to the very steep slopes involved in the input there are times where small infusions of extra glucose would be required given the demand for "negative" insulin in the figure. This limitation of the optimal result can be quantified by the demand for what are essentially insulin injections of 1-3U followed in the two largest cases by small glucose infusions to account for the demand for $0.3 \mathrm{U}$ withdrawals of insulin from the subcutaneous area. The normal insulin response in Figure 6 is shaped very much like the input itself, as would be expected although it appears flat in the figure.

\subsection{Summary of Results}

The optimal controller is seen to provide, with no additional feedback an ideal, flat line glucose response for both a normal ICU feeding profile and an extreme case. The extreme case does lead to potential problems with the demand for negative insulin that would require an additional, uncorrected glucose infusion. However, this case is extreme and not representative of any accepted glucose feeding protocol for critical care patients in either rapidity of infusion, or total amount of calories given. In each case, the normal response was exceeded and tight, nearly perfect glucose control, which is very important for some classes of critical care patients, was maintained.

\section{Conclusions}

The emergence of glucose sensors capable of providing blood glucose readings at a very high rate is investigated along with the ability to automate insulin infusion for diabetic and hyperglycemic ICU patients. ICU patients present a special case where the feeding protocols tend to consist of infrequent, slow or uniform infusions of smaller amounts of glucose. As a result, the glucose input profile is a known and this data can be used to advantage. Although the optimal controller developed does not require feedback of the glucose value these sensors can be used for other forms of feedback as needed and to monitor patient response for safety.

The optimal infusion control law developed is an entirely a function of patient parameters and the glucose input profile. For the slower infusions typically encountered in ICU patients the optimal control law can provide a nearly perfectly flat glucose response with no need for additional glucose infusion for stability. For more extreme tests, such as the multimeal test presented, some glucose infusion is necessary, however the glucose response curve remains flat. In both cases these ideal results outperform normal response, in addition to which ICU patients can often be hyperglycemic due to physiological stress even with no previous diabetic history. Overall, an optimal control law has been developed and tight, nearly ideal glucose regulation shown for ICU scenarios where the glucose input is a known, slow-moving function.

\section{References}

[1] Van den Berghe, G, Wouters, P. Weekers, F., Verwaest, C., Bruyninckx, F., Schietz, M., Vlasselaers, D., Ferdinance, P., Lauwers, P. and Bouillon, R. (2001). "Intensive insulin therapy in critically ill patients," New England J. of Med., vol. 345(19), pp. 1359-1367.

[2] Trajanoski, Z., Jobst, G., Wach, P., Urban, G., Ellmerer, M., Kotanko, P. and Skrabal, F. (1994). "Continuous ex vivo monitoring of glucose in blood and subcutaneous tissue field," Proc. 16th Annual Intl Conf. Of the IEEE Medicine and Biology Society, pp. 814-815.

[3] Trajanoski, Z. and Wach, P. (1995). "Evaluation of the subcuraneous route for closed-loop control of insulin delivery: numerical and experimental studies," Proc. 17th Annual Intl Conf. Of the IEEE Medicine and Biology Society, pp. 1357-1358.

[4] Gfrerer, R. J., Trajanoski, Z., Skrabal, F., Jobst, G., Urban, G., Kotanko, P. and Wach, P. (1996). "Blood withdrawal system for continuous ex vivo glucose and lactate monitoring," Proc. 18th Annual Intl Conf. Of the IEEE Medicine and Biology Society, pp. 75-76.

[5] Alberti KGMM \& Zimmett P Z. Definition, "Diagnosis and classification of diabetes Mellitus - provisional report of a WHO consultation group". Diabetes Med. 1998 15:539-53.

[6] Worthington, D. (1990). "Use of models in the self-management of insulin-dependent diabetes mellitus," Computer Methods and Programs in Biomedicine, vol. 32(3-4), pp. 233-239.

[7] Parker, R., Doyle, F., Harting, J., and Peppas, N. (1996). "Model predictive control for infusion pump insulin delivery," IEEE Conf. Of Engineering in Medicine and Biology, Nov 3, pp. 1822-1823.

[8] Doyle, F., Dorski, C., Harting, J., and Peppas, N. (1995). "Control and modeling of drug delivery devices for the treatment of diabetes," Proc. of 
the American Control Conference, June 21-23, pp. 776-780,

[9] Ollerton, R. (1989). "Application of optimal control theory to diabetes mellitus," Intl. Journal of Control, vol. 50(6), pp. 2503-2522.

[10] Kienitz, K. and Yoneyama, T. (1993). "Robust controller for insulin pumps based on H-infinity theory," IEEE Trans. On Biomedical Engineering, vol. 40(11), pp. 1133-1137.

[11] Fisher, Michael E., (1991). “A Semiclosed-Loop Algorithm for the Control of Blood Glucose Levels in Diabetics", IEEE Transactions on Biomedical Engineering, vol 38, No. 1 pp 57-61

[12] Trajonoski, Z. and Wach, P. (1995). "Evaluation of subcutaneous route for the closed-loop control of insulin delivery: Numerical and experimental studies," IEEE Conf. on Engineering in Medicine and Biology, Sep 2023, pp. 1357-1358.

[13] Cobelli, C., Nucci, G., and Del Prato, S. (1999). "Physiological simulation model of the glucoseinsulin system," IEEE Conf. on Engineering in Medicine and Biology, Oct 16, pp. 999.

[14] Furler, Stuart M., Kraegen, Edward W., Smallwood, Robert H., and Chisholm, Donald J. (1985). "Blood Glucose Control by Intermittent Loop Closure in the Basal Mode: computer Simulation Studies with a Diabetic Model", Diabetes Care, Vol. 8, No. 6 pp 553 - 561.

[15] Bergman, Richard N., Finegood, D. T., and Ader, M., (1985) "Assessment of Insulin Sensitivity in Vivo", Endocrine Reviews, vol 6, no 1 , pp 45- 86 .

[16] Kraegen, E. W. and Chisolm, D. J., (1984), "Insulin responses to carting profiles of subcutaneous insulin infusion: Kinematics modelling studies", Diaetologia, vol. 26, pp 208-213.

[17] Cobelli, C., Federspil, G., Pacibi, G., Salvan, A., and Scandellari, S. (1982) "An Integrated Mathematics Model of the Dynamics of Blood Glucose and Its Hormonal Control" Mathematical Biosciences, vol 58 pp 27 - 60.

[18] Lehman, E. and Deutsch, T. (1996). "Computer assisted diabetes care: a 6-year retrospective," Computer Methods and Programs in Biomedicine, vol 50, pp. 209-230.

[19] Schrezenmeir, J. (1991). "Computer aids in dietetics," Diabetes, Nutrition and Metabolism, Supplement 1, pp. 175-191.

[20] Skyler, J., Skyler, D., Seigler, D. and Sullivan, M. (1981). "Algorithms for adjustment of insulin dosage," Diabetes Care, vol 4, pp. 311318.
[21] Lehman, E. and Deutsch, T. (1993). "AIDA: a mk. II automated insulin dosage advisor," Journal of Biomedical Engineering, vol 15, pp. 201-211.

[22] Boroujerdi, M., Williams, C., Carson, E., Piwernetz, K., Hepp, K., and Sonksen, P. (1987). "A simulation approach for planning insulin regimes," Intl. Symposium on Advanced Models for Therapy of Insulin Dependent Diabetes, pp. 41-46.

[23] Fletcher, L. and Soden, P. (1991). "Diet construction using linear programming," Diabetes, Nutrition and Metabolism, Supplement 1, pp. 169-174.

[24] Berger, M. and Rotbard D. (1991). "A pharmacodynamic approach to optimising insulin therapy," Computer Methods and Programs in Biomedicine, vol 34, pp. 241-253.

[25] Parker, R., Doyle, F., Harting, J. and Peppas, N. (1996). "Model predictive control for infusion pump insulin delivery," IEEE Conf on Eng in Med and Biology, pp. 1822-1823.

[26] Ollerton, R. (1989). "Application of optimal control theory to diabetes mellitus," International Journal of Control, vol 50(6), pp. 2503-2522.

[27] Naylor, J., Hodel, A., Morton, B., and Schumacher, D. (1995). "Automatic control issues in the development of an artificial pancreas," Proc. ACC, pp. 771-775.

[28] Kienitz, K. and Yoneyama, T. (1993). "Robust controller for insulin pumps based on H-infinity theory," IEEE Transactions on Biomedical Engineering, vol 40(11), pp. 1133-1137.

[29] Chase, J. G., Lam, Z-H, Lee, J-Y and Hwang, K-S. (2002). "Active Insulin Control of the Blood Glucose Derivative," Proc. of ICARCV 2002. 